\title{
REFORMA ELECTORAL EN CANARIAS: GÉNESIS, ALCANCE, IMPLEMENTACIÓN E INCIDENCIA PRÁCTICA
}

\author{
VÍCTOR CUESTA LÓPEZ \\ Profesor Contratado Doctor de Derecho Constitucional \\ Universidad de Las Palmas de Gran Canaria
}

TRC, núm. 45, 2020, pp. 429-449

ISSN 1139-5583

\section{SUMARIO}

I. Introducción: una reforma electoral en dos tiempos. II. La ampliación del Parlamento de Canarias. III. Circunscripciones insulares y circunscripción autonómica. IV. El prorrateo de escaños en el régimen electoral transitorio. V. El descenso de las barreras electorales. VI. La implementación de la reforma electoral en las elecciones autonómicas de mayo de 2019. VII. Algunas reflexiones finales sobre la incidencia de la reforma electoral en la práctica.

\section{INTRODUCCIÓN: UNA REFORMA ELECTORAL EN DOS TIEMPOS}

Tras una larga tramitación parlamentaria, que se prolonga entre la $\mathrm{X}$ y la XII legislatura, la reforma del Estatuto de Autonomía de Canarias (en adelante, EACan) es aprobada mediante Ley Orgánica 1/2018, de 5 de noviembre, reuniendo el apoyo de una amplia mayoría de las fuerzas políticas presentes en las Cortes Generales ${ }^{1}$. Esta revisión estatutaria, de carácter integral, afecta a las bases

1 En la sesión plenaria del Congreso de los Diputados celebrada el 13 de septiembre de 2018, el dictamen de la Comisión Constitucional del Congreso de los Diputados contó con el voto favorable de 237 diputados (integrados en los grupos parlamentarios Popular, Socialista, nacionalistas canarios, PDeCAT y PNV), con el voto en contra de 60 diputados (del Grupo Parlamentario Confederal de Unidos Podemos) y con la abstención de 39 diputados (Ciudadanos, Esquerra Republicana de Catalunya). En la sesión plenaria celebrada en el Senado el 24 de octubre de 2018, se rechazan todas las enmiendas que se habían mantenido, y efectuada la votación sobre el conjunto del texto, se da el siguiente resultado: senadores presentes, 244; votos emitidos, 235; a favor, 214; en contra, 18; abstenciones, 3. 
del particular sistema electoral canario cuya reforma se había demandado de manera insistente desde la doctrina ${ }^{2}$ y desde amplios sectores de la clase política y la sociedad civil canaria. Conviene advertir, no obstante, que el alcance de la reforma electoral no fue el que inicialmente había previsto el Parlamento de Canarias en la propuesta de revisión del EACan aprobada en marzo de 2015. El legislador estatuario autonómico apuesta en ese momento por una modificación más limitada de la que resultó tras su paso por la Comisión Constitucional del Congreso de los Diputados y que se inspiraba en la que ya se había planteado en la propuesta de reforma estatutaria remitida a las Cortes Generales en el año 2006 pero que finalmente fue retirada por el propio Parlamento de Canarias.

Entre las principales novedades en materia electoral contenidas en la propuesta de reforma estatutaria aprobada por el Parlamento de Canarias destaca la posible creación de una nueva circunscripción de ámbito autonómico que podría coexistir con las tradicionales circunscripciones insulares y que se condicionaba, sin embargo, a lo que en el futuro pudiese establecer la ley reguladora del régimen electoral. Esta ley electoral, que ahora podría ser aprobada por la mayoría cualificada de tres quintos — sensiblemente inferior a la mayoría de dos tercios que el EACan anterior requería para la aprobación de la ley que modificase los elementos del sistema electoral determinados por su Disposición Transitoria Primera (en adelante, DTP $)^{3}$ - , también debía integrar un prorrateo de escaños que no podría suponer la asignación a una circunscripción insular de «un número de diputados inferior a otra que tenga menos población de derecho». No obstante, la DTP de la propuesta de reforma del EACan aprobada por el Parlamento de Canarias contravenía abiertamente este mandato manteniendo el mismo prorrateo de escaños entre circunscripciones insulares asignando, de modo directo, 7 diputados a la

2 Sirva como ejemplo, entre la doctrina más autorizada, la opinión de López Aguilar: «no hay comunidad autónoma en la que el sistema electoral haya producido mayor perjuicio al crédito del autogobierno y por tanto haya contribuido más a la deslegitimización de la experiencia autonómica que Canarias. El sistema electoral canario no solamente fragmenta el paisaje político, sino que perjudica el crédito de la política y ha deteriorado, como en ninguna otra comunidad autónoma, la confianza del elector de que con su voto contribuye a la formación de gobierno o por lo menos a deshacerse del gobierno que no le gusta». López Aguilar, J. F., «El Sistema electoral autonómico canario: una revisión necesaria, imperiosa e inaplazable», en PÉrEZ SÁNChez, G. - Mujica Moreno, V. (coords.), Textos para la reforma electoral de Canarias, Las Palmas de Gran Canaria: Universidad de Las Palmas de Gran Canaria, 2016, pp. 156-157.

3 El Estatuto anterior remitía a una ley que debía ser aprobada por mayoría de 2/3 para regular los elementos del sistema electoral que supusieran una modificación de lo establecido en la DTP EACan. El Dictamen del Consejo Consultivo de Canarias 540/2018, de 24 de noviembre de 2018, explica claramente esta cuestión: «Antes había una reserva parcial a una Ley aprobada por mayoría de dos tercios, mayoría que solo era necesaria (y que nunca se aprobó) para cambiar la distribución de escaños y las magnitudes de las barreras electorales establecidas en la anterior Disposición transitoria primera, pues expresamente establecía en ambos apartados que «en tanto no se disponga otra cosa por una Ley del Parlamento Canario aprobada por mayoría de dos terceras partes de sus miembros» se fijaba la distribución de escaños y las barreras electorales. El resto de cuestiones, reguladas por la actual Ley 7/2003, de 20 de marzo, de Elecciones al Parlamento de Canarias, únicamente requería mayoría simple», Dictamen del Consejo Consultivo de Canarias 540/2018, de 28 de noviembre, fundamento IV.4. 
circunscripción de Fuerteventura, isla que, como comprobaremos en los próximos apartados, contaba con más población de derecho que La Palma, a la que asignaba 8 escaños. Sin lugar a dudas, la apuesta más decidida del Parlamento de Canarias, y la que suscitó en todo momento mayor consenso político, fue la drástica reducción, a la mitad, de las dobles barreras electorales que se habían incrementado notablemente tras la anterior reforma estatutaria de 1996. De este modo, en las circunscripciones insulares sólo serían tenidas en cuenta las listas de partido o coalición que hubiesen superado el 15 por ciento de los votos válidos emitidos en esa circunscripción, o, de manera alternativa, las listas de aquellos partidos o coaliciones que, sumando los votos reunidos en todas las circunscripciones insulares, hubiesen superado el 3 por ciento del total de los votos válidos emitidos en el conjunto del archipiélago.

Considerando la dificultad en sumar al acuerdo de reforma a las fuerzas tradicionalmente beneficiadas por este complejo sistema electoral y el fracaso de todas las propuestas que se venían presentando periódicamente $e^{4}$, las modificaciones acordadas por el Parlamento de Canarias podían llegar a mostrarse ante la opinión pública como un avance muy relevante. Sin embargo, las elecciones autonómicas celebradas en mayo de 2015 vuelven a arrojar unos resultados tremendamente

4 En el Dictamen de la Comisión de Estudio sobre la reforma electoral constituida en el seno del Parlamento de Canarias, aprobado el 28 de mayo de 2018, se presenta un amplio resumen de las distintas propuestas de reforma que habían sido presentadas a partir de 1996: «los intentos de modificar el sistema electoral han sido varios. El primero de ellos tuvo lugar en el año 2000, cuando dos diputados de AHI integrados en el Grupo Mixto presentaron una Proposición de Ley cuyo propósito era aumentar en 6 el n ${ }^{\circ}$ de diputados de la Cámara, alcanzando un total de 66 escaños. Para ello, se adjudicaba esos nuevos escaños de forma equitativa entre las circunscripciones de Tenerife y Gran Canaria, de forma que se disminuyese la infrarrepresentación de estos territorios. Asimismo, la propuesta pretendía eliminar la barrera autonómica del 6 por ciento, así como reducir la barrera insular al 15 por ciento. Sin embargo, este intento se vio frustrado por una enmienda a la totalidad introducida por el Grupo Nacionalista Canario, que dejó las cosas como estaban. La segunda de las tentativas tuvo como protagonista al Grupo Parlamentario Socialista, que en el año 2009 trató de sacar adelante una Proposición de Ley para la «Democratización del sistema electoral al Parlamento de Canarias». Esta propuesta consistía en eliminar la barrera autonómica del 6 por ciento y fijar una única barrera electoral en el 5 por ciento insular. En la exposición de motivos se argumentaba que esta medida no era todo lo ambiciosa que podía ser, pues no alteraba cuestiones como el $\mathrm{n}^{\circ}$ de diputados del Parlamento, la distribución del $\mathrm{n}^{\circ}$ de representantes entre las islas, o la creación de una circunscripción adicional de ámbito autonómico. Según sus proponentes, era una oferta ideada para concitar el consenso del resto de formaciones parlamentarias. No obstante, aunque dicha propuesta fue tomada en consideración por el Pleno del Parlamento en octubre de 2009 y continuó su tramitación en ponencia y en comisión sin enmienda alguna, terminó caducando al finalizar la legislatura, sin que fuese finalmente elevada al Pleno de la Cámara para su aprobación final. El intento más reciente se produjo en el año 2013, momento en el que Nueva Canarias (NC), cuyos tres diputados se encontraban por entonces integrados en el Grupo Mixto, planteó una reforma electoral exactamente idéntica a la que propusiera el PSC-PSOE en el año 2009 (eliminar la barrera electoral regional y fijar la insular en el 5 por ciento). Dicha proposición fue desechada gracias a los votos en contra de la mayoría parlamentaria que sustentaba al Ejecutivo de coalición entre CC y PSOE. Estos partidos argumentaban que los cambios del sistema electoral debían producirse en el seno de la reforma del EA canario que comenzó su tramitación a principios de 2014, Dictamen de la Comisión de estudio sobre la reforma del sistema electoral canario», Dictamen de la Comisión de estudio del Parlamento de Canarias 9L/AGCE-0005, Sobre la reforma del sistema electoral canario, publicado en el BOPC, IX legislatura, Año 2018, No 206, 21 de mayo, p. 16. 
injustos que derivan, principalmente, de la enorme descompensación del valor del voto entre las circunscripciones insulares sobrerrepresentadas (las islas no capitalinas) y las infrarrepresentadas (Tenerife y Gran Canaria) que resulta, a su vez, del prorrateo de escaños conforme a la regla de la triple paridad y de la aplicación de las dobles barreras electorales. Sirva como ejemplo ilustrativo el caso de Coalición Canaria, el partido que situándose en tercera posición en número de votos $(17,92 \%$ de los votos válidos emitidos en el conjunto del archipiélago) obtiene más escaños (18) que el partido más votado (el Partido Socialista con un 19,53\% de los votos obtiene 15 escaños). También resulta significativo que Ciudadanos-Partido de la Ciudadanía, con 54.375 votos que representan un 5,84\% de los votos emitidos en la Comunidad Autónoma, no obtiene representación (ya que no supera la barrera electoral del $6 \%$ de votos válidos emitidos en el conjunto de todas las circunscripciones insulares) mientras que la Agrupación Socialista Gomera, con 5.090 votos que representan el $0,55 \%$ de los votos emitidos en el conjunto del archipiélago, obtiene 3 escaños en la circunscripción electoral de La Gomera, donde concentra todos sus sufragios, un $42 \%$ del total de votos emitidos en la isla.

Estos resultados evidencian, una vez más, graves incongruencias que no iban a ser solventadas con las modificaciones planteadas por la propuesta de reforma del Parlamento de Canarias. En este contexto poselectoral se multiplican las voces que piden una reforma de mayor calado. Merece ser destacada la labor que realiza en este sentido Demócratas para el Cambio, una plataforma ciudadana sin adscripción política, constituida desde 2007, que a través de actividades divulgativas y distintas campañas informativas venía promoviendo el debate en torno a esta problemática ${ }^{5}$. En junio de 2015, Demócratas para el Cambio consigue reu-

5 El presidente de esta asociación nos ofrece un buen repaso de las distintas acciones impulsadas en Mujica Moreno, V., «Crónica de la reclamación de la reforma electoral desde la sociedad civil: Demócratas para el Cambio», en Pérez Sánchez G. - Mujica Moreno V. (coords.), Textos para la reforma electoral de Canarias, op. cit., pp. 204-272. La labor de Demócratas para el Cambio también es destacada en el Dictamen de la Comisión de estudio del Parlamento de Canarias sobre la reforma electoral: «Resulta pertinente hacer mención al foro cívico Demócratas para el Cambio (DPC). Esta plataforma, nacida de la sociedad civil, viene reclamando una profundización democrática a través de la reforma del sistema electoral canario, con el objetivo de que dicho sistema sea más proporcional, más igualitario y menos restrictivo, en lo que constituye un ejemplo de participación ciudadana sin precedentes en Canarias. Su trayectoria de reivindicación cívica se remonta hasta el año 2007. En junio de 2014, DPC propuso a las organizaciones políticas de las islas un compromiso para acordar tres puntos básicos sobre los que empezar a construir un consenso para alcanzar una nueva norma electoral canaria. Se trataba del documento Pacto por la Democracia en Canarias que básicamente proponía asumir tres puntos: 1) Reducir las barreras electorales (sin precisar su n ni su cuantía); 2) Mejorar la proporcionalidad (sin precisar la forma de hacerlo); y 3) Que la nueva norma electoral esté vigente antes de la convocatoria de elecciones autonómicas de 2019. Este compromiso de mínimos fue asumido por doce organizaciones políticas a las que en 2016 se sumaron Podemos y PP, quedando ausentes únicamente CC y PSOE, partidos que por aquel entonces aún conformaban una coalición de gobierno. Tampoco fue rubricado por la Agrupación Socialista Gomera (ASG). El papel proactivo de la sociedad civil organizada a través de DPC ha sido un elemento clave en la dinamización del debate en la sociedad canaria y en la búsqueda de soluciones para la materia de la que se encarga esta comisión de estudio», Dictamen de la Comisión de estudio del Parlamento de Canarias 9L/AGCE-0005, Sobre la reforma del sistema electoral canario, publicado en el BOPA, IX legislatura, 2018, $\mathrm{n}^{\circ}$ 206, 21 de mayo, p. 16. 
nir el apoyo de hasta 75 organizaciones de la sociedad civil y partidos políticos de Canarias al manifiesto Reforma Electoral Canaria ¡Ya!, defendido también en las calles de las capitales canarias por miles de personas, y que denunciaba claramente «la elevada restricción de acceso al escaño y la enorme desigualdad de valor de voto entre sus ciudadanos» ${ }^{6}$. Los partidos políticos que obtienen representación en el Parlamento de Canarias después de las elecciones de mayo de 2015 también parecen asumir la necesidad de avanzar en la reforma electoral. Recién abierta la IX legislatura, los grupos parlamentarios Popular, Nueva Canarias y Podemos solicitan la creación de una Comisión de estudio sobre la reforma del sistema electoral canario, aunque hubo que esperar a enero de 2016 para que ésta finalmente se constituyese, a iniciativa de los grupos parlamentarios Nacionalista y Socialista que apoyaban al gobierno de coalición. En este momento, también va cobrando fuerza la idea de que los preceptos relativos al sistema electoral canario incluidos en la propuesta de reforma del EACan podrían ser modificados durante la tramitación parlamentaria pendiente en las Cortes Generales ${ }^{7}$.

Los trabajos de la Comisión de estudio del Parlamento de Canarias se extienden entre abril de 2016 y mayo de 2018. Tras consultar a representantes de partidos políticos extraparlamentarios, miembros de la sociedad civil y expertos en materia electoral, la Comisión aprueba, por una amplia mayoría que supera el significativo umbral de los tres quintos, el Dictamen que incluye una propuesta de reforma electoral con novedades significativas respecto a la propuesta de reforma del EACan aprobada en 2015. Evidentemente, este Dictamen no vinculaba jurídicamente a las Cortes Generales que en ese momento estaban tramitando la reforma estatutaria, pero sí podría ser indicativo de los cambios que el Parlamento de Canarias podría aceptar en caso de que se produjese una modificación sustancial respecto de la propuesta originaria. El Dictamen de la Comisión de estudio propone un régimen electoral transitorio que contemple la ampliación del Parlamento de Canarias de sesenta a setenta escaños y, partiendo de la triple paridad, la asignación de un escaño adicional a Fuerteventura, asumiendo de este modo el principio de que ninguna circunscripción insular podía tener asignados un número de diputados inferior al de otra circunscripción insular con menos

6 Manifiesto Reforma Electoral Canaria ;Ya!, publicado en la web de la asociación: (https://democratasparaelcambio.com/2015/07/18 texto-del-manifiesto-reforma-electoral-canaria-ya-\%E2\%80\% A2-movilizaciones-del-19-de-junio-de-2015/)

7 Los trabajos de la Comisión de estudio transcurren en paralelo a la tramitación de la propuesta del EA de Canarias en la Comisión Constitucional del Congreso de los Diputados. Como se explica claramente en el Dictamen de la Comisión de estudio del Parlamento de Canarias, «en cierto modo, la segunda apertura de comisión de estudio presentada por los grupos que apoyaban al gobierno, perseguía que los debates de la comisión no transcurrieran de espaldas a las novedades que en materia electoral preveía el texto estatutario enviado al Congreso de los Diputados. (...) Precisamente, el motivo de las sucesivas prórrogas ha sido la de esperar por las conclusiones de esta comisión y su Dictamen para tener en cuenta la opinión del Parlamento de Canarias sobre la reforma de su sistema electoral», Dictamen de la Comisión de estudio del Parlamento de Canarias 9L/AGCE-0005, Sobre la reforma del sistema electoral canario, publicado en el BOPA, IX legislatura, 2018, n' 206, 21 de mayo, pp. 17-18. 
población de derecho. Los nueve escaños adicionales restantes se asignarían directamente a la circunscripción autonómica sin tener que esperar a lo que pudiese determinar en el futuro la ley de régimen electoral. Por otra parte, la barrera electoral que debería operar en el conjunto de las circunscripciones insulares, y también en la nueva circunscripción autonómica, se fija en el $4 \%$ de los votos emitidos en toda la Comunidad Autónoma, un punto más de lo previsto en la propuesta de reforma de EACan aprobada en 2015. Finalmente, el Dictamen de la Comisión de estudio recoge «la aspiración y compromiso conjunto de los cuatro grupos parlamentarios Socialista, Popular, Podemos y Nueva Canarias y de las organizaciones políticas que representamos, de promover las iniciativas legislativas necesarias para lograr esta modificación del sistema electoral y que esté vigente para la convocatoria de las elecciones de 2019» ${ }^{8}$.

Mientras tanto, la ponencia constituida en la Comisión Constitucional del Congreso de los Diputados trabajaba en el informe sobre la Propuesta de reforma del EACan'. Es en el seno de esta ponencia en el que se aprueban dos enmiendas transaccionales, una al art. 37 y otra a la DTP, que habían sido pactadas entre los diputados del PSOE y Nueva Canarias, fuerzas políticas que también se habían sumado al acuerdo alcanzado en la Comisión de estudio del Parlamento de Canarias $^{10}$. No es de extrañar, por tanto, que la nueva redacción que se da a estos dos artículos coincida con la propuesta de reforma electoral contenida en el Dictamen de la Comisión de estudio que acabamos de presentar. Debemos destacar, no obstante, dos novedades significativas: el número máximo de diputados que podría alcanzar el Parlamento de Canarias, conforme a lo que establezca en el futuro la ley reguladora del régimen electoral, se incrementa hasta setenta y cinco. Por otra parte, la ponencia también incorpora una enmienda a la DTP, presentada por el Grupo Parlamentario Confederal de Unidos Podemos - En Comú Podem-. En Marea, que supone la adición del apartado cuarto en el que establece un plazo de tres años, desde la entrada en vigor del EACan, para que el Parlamento de Canarias elabore la ley que debe regular el régimen electoral.

Una vez aprobado el Dictamen de la Comisión Constitucional en la sesión plenaria del Congreso de los Diputados de 13 de septiembre de 2018, la propuesta

8 Dictamen de la Comisión de estudio del Parlamento de Canarias 9L/AGCE-0005, Sobre la reforma del sistema electoral canario, publicado en el BOPA, IX legislatura, Año 2018, No 206, 21 de mayo, p. 19.

9 La Ponencia encargada de redactar el Informe sobre la Propuesta de reforma del EA de Canarias estaba integrada por los Diputados M.C. Hernández Bento (Grupo Popular), G. Mariscal Anaya (Grupo Popular), P. Matos Mascareño (Grupo Popular), G. Cámara Villar (Grupo Socialista), S. Franquis Vera (Grupo Socialista), M.C. Pita Cárdenes (Grupo Confederal Unidos Podemos-En Comú Podem-En Marea), A. Rodríguez Rodríguez (Grupo Confederal Unidos Podemos-En Comú Podem-En Marea), S. Ramírez Freire (Grupo Ciudadanos), M. Rodríguez Hernández (Grupo Ciudadanos), M. Legarda Uriarte (Grupo Vasco-EAJ-PNV), A.M. Oramas González-Moro (Grupo Mixto) y P. Quevedo Iturbe (Grupo Mixto).

$10 \mathrm{La}$ enmienda transaccional al artículo 37 integraba las enmiendas 188 del Grupo Parlamentario Mixto (Sr. Quevedo Iturbe) y 287 del Grupo Parlamentario Socialista. La enmienda transaccional a la disposición transitoria primera integraba la enmienda 189 del Grupo Parlamentario Mixto (Quevedo Iturbe) y 320 del Grupo Parlamentario Socialista. 
de reforma del EACan se dirige al Senado que, a la postre, no modifica ninguno de sus artículos. No obstante, y considerando que la propuesta de reforma había sido modificada a su paso por el Congreso, el Senado la remite antes al Parlamento de Canarias cumpliendo con lo dispuesto en el art. 64.2 del EACan todavía vigente en aquél momento: «Si las Cortes Generales, durante la tramitación parlamentaria, modificaran sustancialmente la reforma propuesta, se devolverá al Parlamento de Canarias para nueva deliberación, acompañando mensaje motivado sobre el punto o puntos que hubieren ocasionado su devolución y proponiendo soluciones alternativas, en cuyo caso el Parlamento de Canarias podrá acceder a las mismas, proponer otras soluciones o desistir de la reforma estatutaria». En el informe emitido el 10 de octubre de 2018, el Parlamento de Canarias constata la existencia de modificaciones sustanciales, también en materia electoral, pero considera que las diferencias que «existen frente a la nueva redacción del artículo 39 y la disposición transitoria primera, no obstan para constatar que la Cámara expresó una posición mayoritaria favorable a la misma con la aprobación del dictamen de la comisión de estudio constituida para la reforma electoral». En consecuencia, el Parlamento de Canarias acuerda acceder a las modificaciones producidas en la propuesta de reforma del Estatuto de Autonomía de Canarias.

En los siguientes apartados del presente trabajo analizaremos los importantes cambios en el régimen jurídico de las elecciones al Parlamento de Canarias tras la entrada en vigor de la reforma del EACan, incidiendo particularmente en la ampliación del Parlamento de Canarias, la creación de la circunscripción de ámbito autonómico, el prorrateo de escaños entre las circunscripciones insulares y la circunscripción autonómica y la reducción de las barreras electorales. Considerando que las nuevas disposiciones estatutarias (art. 39 y DTP EACan) debían ser aplicadas necesariamente en las elecciones autonómicas celebradas en mayo de 2019, también ofreceremos un análisis de los instrumentos jurídicos que, a falta de una ley electoral, fueron utilizados para su implementación. Finalmente, y atendiendo a los resultados de las citadas elecciones, presentaremos algunas reflexiones en relación a la incidencia práctica de la reforma electoral canaria.

\section{LA AMPLIACIÓN DEL PARLAMENTO DE CANARIAS}

El art. 39.2.b) EACan determina que el número de diputados que integran el Parlamento de Canarias debe oscilar entre cincuenta y setenta y cinco, aumentando esta última cifra máxima en cinco respecto a lo previsto antes de la reforma de 2018. Asimismo, y mientras no se aprueba la ley que debe regular el régimen electoral fijando un número concreto, la DTP determina que el número de diputados asciende a setenta, diez más que lo dispuesto por el régimen transitorio anterior. Este incremento es bastante significativo, en términos cualitativos, si tenemos en cuenta que las últimas reformas electorales en nuestro Estado autonómico, en un contexto económico muy complicado, habían apostado por la reducción de las 
Asambleas Legislativas. Como nos advierte García Mahamut en su estudio sobre la reforma de los sistemas electorales autonómicos, «bajo el eslogan «más austeridad y reducción del déficit público», con la mayoría absoluta del PP, algunas Asambleas Legislativa reformaron sus respectivas leyes electorales y/o Estatutos para rebajar el número de diputados que componían la Cámara. La reforma de mayor enjundia fue la que se llevó a cabo en Castilla-La Mancha en 2014. Con el apoyo de la mayoría absoluta del PP, y con la oposición parlamentaria en contra, se reformó el Estatuto de Autonomía y la ley electoral para, entre otras medidas, disminuir drásticamente el número de escaño pasando éste de 53 a 33 diputados» ${ }^{11}$.

No es el objeto de este apartado valorar cuál debería ser la dimensión óptima de una asamblea legislativa que aspira a representar a una comunidad política compleja y plural (como lo es, sin duda, la comunidad autónoma de Canarias), pero sí deberíamos relativizar las críticas que, desde una óptica exclusivamente economicista, denuncian que el incremento del gasto público que deriva de la ampliación está totalmente injustificado. En primer lugar, el Parlamento de Canarias después de esta ampliación sigue siendo una asamblea legislativa relativamente reducida ${ }^{12}$. Con una población de derecho que, a 1 de enero de 2018, asciende a 2.127.685 personas, la ratio de habitantes por escaño es 30.395,5, todavía superior a la de la mayoría de asambleas legislativas autonómicas. No obstante, tras la implementación de la reforma estatutaria de 2018, lo cierto es que esta ratio se sitúa por debajo de la media del conjunto de las CCAA $(33.601,39$ habitantes por escaño $)^{13}$.

En cualquier caso, debemos tener en cuenta que el principal déficit del sistema electoral canario era, y posiblemente todavía sigue siendo después de la reforma estatutaria, la enorme descompensación del valor relativo del voto entre

11 García Mahamut, R., «La reforma de los sistemas electorales autonómicos tras las elecciones de 24 de mayo de 2015: vectores políticos y jurídicos», TRC, núm. 41, 2018, p. 189.

12 Si la comparación se establece con las asambleas legislativas de los archipiélagos de Baleares, Azores y Cabo Verde, el Parlamento de Canarias, antes de la reforma de 2018, resulta ser la asamblea legislativa más reducida: «el Parlamento de Canarias es bastante más reducido en términos relativos ya que, con el censo electoral más numeroso, la media de electores por cada uno de los 60 escaños asciende a 27.688, cifra que llega a ser más del doble que en el caso del parlamento balear ( 1 escaño - 12.625 electores). La diferencia en términos relativos con Cabo Verde (media de 4.147 electores por escaño) y Azores (media de 3.926 electores por escaño) es aún mucho mayor: la media del Parlamento de Canarias llega a ser siete veces superior respecto a la de la asamblea legislativa del archipiélago portugués», CUESTA LóPEZ, V., «Los sistemas electorales archipielágicos: reflexiones en torno a la reforma del sistema electoral canario desde el derecho comparado», en PÉREZ SÁNChez, G. y Mújica Moreno, V. (coords.), Textos para la reforma electoral de Canarias, op. cit., pp. 116-117.

13 Según el Instituto Canario de Estadística, y en atención a las cifras de población correspondiente a 2018, la ratio de habitantes por escaño en las Asambleas legislativas autonómicas, de menor a mayor, es la siguiente: La Rioja (9.566); Comunidad Foral de Navarra (12.951); Extremadura (16.506); Cantabria (16.578); Illes Balears (19.134); Aragón (19.533); Principado de Asturias (22.850); País Vasco (29.321); Castilla y León (29.743); Canarias tras la reforma estatutaria de 2018 (30.396); Región de Murcia (32.856); Canarias con el Estatuto de Autonomía original de 1982 (35.461); Galicia (36.023); Comunitat Valenciana (50.138); Comunidad de Madrid (50.993); Cataluña (56.297); Castilla-La Mancha (61.418); Andalucía (76.921). 
sus circunscripciones. Si se pretendía atenuar este desequilibrio manteniendo el número de escaños en sesenta, hubiese sido necesario reducir el número de escaños que se asignaban a las circunscripciones sobrerrepresentadas, las islas no capitalinas, medida que hubiese complicado, o tal vez imposibilitado, el acuerdo político. Por tanto, la ampliación del Parlamento se presentaba como la opción más plausible para atenuar esta descompensación, si bien, como veremos en los próximos apartados, el legislador estatutario no opta por asignar los escaños adicionales a las circunscripciones insulares infrarrepresentadas, Gran Canaria y Tenerife, sino, en su gran mayoría (nueve de diez) a la nueva circunscripción autonómica.

También debemos constatar que, aunque para hacer frente a las críticas que cuestionan la ampliación del Parlamento de Canarias por razones presupuestarias, los grupos parlamentarios Socialista, Popular, Podemos y Nueva Canarias se comprometían en el Dictamen de la Comisión de estudio sobre la reforma electoral «a llevarla a cabo sin incremento del gasto global del presupuesto del Parlamento de Canarias correspondiente a la actuación legislativa y de control», lo cierto es que el gasto en este capítulo sí se ha incrementado sensiblemente. Conforme a los datos ofrecidos por la propia Cámara, los gastos correspondientes a la «Actuación legislativa y de control», incluidos en el capítulo «Servicios Generales», se incrementaron entre 2017 y 2018 desde los 15.947.700€ a los 16.222.930€, y también entre 2018 y 2019, llegando este último año a los $20.230 .814 €^{14}$.

\section{CIRCUNSCRIPCIONES INSULARES Y CIRCUNSCRIPCIÓN AUTONÓMICA}

El art. 39.2.c) EACan contempla la posibilidad de que en el sistema electoral canario coexistan dos tipos de circunscripciones con distinto ámbito territorial: las circunscripciones insulares y la circunscripción autonómica que se extiende al conjunto del archipiélago. Aunque, empleando una redacción bastante confusa, el primer enunciado de este precepto parece dar a entender que el legislador podría optar por uno de los dos tipos de circunscripciones o por compaginar ambas ( «las circunscripciones electorales podrán ser de ámbito autonómico, insular o de ambas»), lo cierto es que, a continuación, es el propio EACan el que constituye las siete circunscripciones insulares correspondientes a cada una de las islas con administración propia $^{15}$. Se cumple así con el mandato previsto en el art. 152 CE sobre la necesaria representación de las diversas zonas del territorio en las asambleas legislativas

14 Datos ofrecidos por el Parlamento de Canarias en su web (https://www.parcan.es/transparencia/ presupuestos/)

15 Las siete islas con administración propia son El Hierro, Fuerteventura, Gran Canaria, La Gomera, Lanzarote, La Palma y Tenerife (Art. 4.1 EACan). La isla de La Graciosa no cuenta con administración propia y a efectos electorales queda incluida en la circunscripción insular de Lanzarote. 
autonómicas. La insularidad, como hecho geográfico objetivo, se impone sobre cualquier otro criterio histórico o político-administrativo para hacer de la circunscripción insular la unidad territorial central del sistema electoral canario. Además, debemos tener en cuenta que en su Sentencia 225/1998, el Tribunal Constitucional ya afirmaba que la elección de las circunscripciones electorales insulares, aunque opera «como criterio parcialmente corrector de la proporcionalidad», resulta «constitucionalmente lícita en la medida en que la misma encuentra apoyo en el denominado «hecho insular», circunstancia geográfica específica, propia de las Comunidades Autónomas que son territorialmente un archipiélago, y cuya singularidad es tenida en cuenta por la propia Constitución (artículos 69.3 y 141.4)» ${ }^{16}$.

Mientras que el art. 39.2.c) EACan constituye directamente las siete circunscripciones insulares, la creación de la circunscripción autonómica parece condicionarse, en cambio, a la voluntad del legislador ordinario. La ley que venga a regular el régimen electoral canario podrá, o no, asignar escaños a esta circunscripción electoral fijando, en su caso, posibles barreras electorales. No obstante, y mientras no se apruebe esta norma, la DTP del EACan determina que a la circunscripción autonómica le corresponden nueve escaños. Esta previsión, además, debía aplicarse necesariamente en las primeras elecciones autonómicas celebradas a partir de la entrada en vigor del EACan, previstas para mayo de 2019. La coexistencia de estos dos tipos de circunscripciones electorales ha supuesto una novedad destacada en el panorama de nuestro derecho electoral autonómico, aunque lo cierto es que sí encontramos algún precedente cercano en derecho comparado. Especialmente significativo, por tratarse también de un archipiélago atlántico, es el caso de Azores, que además de una circunscripción electoral en cada isla cuenta con una circunscripción de ámbito archipielágico. En este caso, se trata de una circunscripción electoral de compensación a la que se asignan cinco escaños que no se reparten entre las listas en función de los sufragios obtenidos sino en función de los votos emitidos en las circunscripciones insulares y que no han servido para obtener representación ${ }^{17}$.

La creación de la circunscripción autonómica atenúa la descompensación del valor relativo del voto que resulta del prorrateo de escaños entre las circunscripciones insulares desde el momento en que los sufragios para la elección de los diputados que se asignan a la circunscripción autonómica se concentran especialmente en

16 STC 225/1998, de 25 de noviembre, F.J. $7^{\circ}$.

17 La circunscripción regional en Azores «se introduce tras la reforma de la legislación electoral de 2006 (Ley orgánica 5/2006) que, entre otros objetivos, pretendía reforzar la proporcionalidad y corregir de algún modo la sobrerrepresentación de las islas con menor $n^{\circ}$ de electores. La solución encontrada es la creación de una circunscripción regional a la que se asignan 5 diputados y que es de carácter adicional y complementario. Esta circunscripción pretende corregir eventuales distorsiones y compensar, especialmente, a los partidos políticos que hayan sido perjudicados en el escrutinio de las circunscripciones insulares aprovechando los votos excedentes que no han servido para obtener representación. De este modo, la elección de los cinco diputados de la circunscripción regional tiene carácter indirecto y deriva de los votos emitidos por los ciudadanos electores en cada una de las islas. También indirecta es la conformación de la candidatura a la circunscripción regional que deriva de las listas propuestas por los diferentes partidos en las circunscripciones insulares», Cuesta López, V., op. cit., p. 119. 
las circunscripciones de Tenerife y Gran Canaria. En este sentido, Fernández Esquer también considera que con la circunscripción electoral autonómica «se mejora la ratio diputado/habitante, se disminuyen las enormes diferencias en el valor del voto existente entre electores de distintas islas y, además, se aumenta la proporcionalidad del sistema» ${ }^{18}$. Más allá de los efectos directos en el conjunto del sistema electoral canario, la creación de la circunscripción autonómica puede favorecer la difusión de un discurso político de ámbito regional, aunque esto dependerá, lógicamente, de la campaña que decidan emprender los partidos que presenten listas en esa circunscripción. Además, si bien es cierto que el EACan nada dice respecto a la circunscripción de la que debe provenir el diputado que quiera presentarse como candidato a la Presidencia de Canarias (art. 48.1 EACan), es posible que buena parte de los partidos políticos canarios terminen situando a sus aspirantes en la cabeza de la lista autonómica facilitando, de este modo, su proyección política en todo el archipiélago.

\section{EL PRORRATEO DE ESCAÑOS EN EL RÉGIMEN ELECTORAL TRANSITORIO}

El prorrateo de escaños entre las circunscripciones electorales insulares en Canarias había seguido desde las primeras elecciones autonómicas la conocida regla de la triple paridad, un compromiso político del estatuyente que respondía a circunstancias histórico-políticas particulares, claramente marcadas por el pleito insular. De la asignación directa de escaños que se hacía desde la DTP del EACan aprobado en 1982 a cada una de las circunscripciones insulares (Gran Canaria y Tenerife, quince escaños; La Palma y Lanzarote, ocho escaños; Fuerteventura, siete escaños; La Gomera, cuatro escaños; El Hierro, tres escaños) resultaba que las circunscripciones insulares de cada una de las dos provincias, Las Palmas y Santa Cruz de Tenerife, sumaban treinta escaños; que las circunscripciones de las dos islas capitalinas sumaban la misma cantidad de escaños, treinta, que las circunscripciones del resto de islas; y que la circunscripción de Gran Canaria y de Tenerife, tenían asignadas cada una quince escaños.

La voluntad del legislador estatuyente de salvaguardar a toda costa estos equilibrios interinsulares, unida a la asimétrica distribución de la población entre las islas que se caracteriza por una mayor concentración de la población en las dos islas capitalinas, ha tenido como consecuencia directa la infrarrepresentación de éstas y la sobrerrepresentación de las circunscripciones insulares menos habitadas. Es cierto que la representación desproporcional entre circunscripciones y la

18 Fernández Esquer, C., «El nuevo sistema electoral de Canarias: un peculiar proceso reformista», Agenda Pública, 4 de noviembre de 2018. Disponible en la web: http://agendapublica.elpais.com/ el-nuevo-sistema-electoral-de-canarias-un-peculiar-proceso-reformista/. 
consiguiente desigualdad relativa del voto (malapportionment) no es una característica exclusiva del sistema electoral canario, pero alcanzaba en éste, utilizando las palabras de López Aguilar, «niveles escandalosos» ${ }^{19}$. En efecto, las circunscripciones insulares de Gran Canaria y Tenerife, que concentran al $82,31 \%$ de la población, sólo tenían asignadas la mitad de los sesenta escaños. Si tomamos como referencia el número de habitantes empadronados en 2018 en la circunscripción de Tenerife, la más poblada con 904.713 habitantes, y el de la circunscripción menos habitada, El Hierro con 10.798 habitantes, y los dividimos por el número de escaños asignados nos encontramos con una ratio de 3.599 habitantes por escaño en El Hierro, frente a 60.314 habitantes por escaño en Tenerife, lo que implica que el valor relativo del voto en la isla de El Hierro es 16,75 veces superior al del voto emitido en Tenerife ${ }^{20}$. Sin embargo, el Tribunal Constitucional, al referirse al sistema electoral de las Illes Balears, afirmaba que sólo considera que la distribución de escaños vulnera el principio de la igualdad del voto cuando la desproporción es al mismo tiempo manifiesta (es decir, de dimensiones considerables) y arbitraria (esto es, sin un criterio objetivo o razonable que la justifique). A sensu contrario, viene a aceptar las diferencias del valor del voto en aquellos supuestos, como el balear, que se justifican por posibilitar la representación adecuada a de cada una de las islas o el mantenimiento de los equilibrios entre las diferentes circunscripciones ${ }^{21}$.

El prorrateo de escaños a las circunscripciones insulares que se propuso desde la DTP del AECan es directo y, por tanto, no preveía su actualización conforme a la evolución demográfica del archipiélago. De este modo, también se daba la paradoja de que la circunscripción insular de Fuerteventura que había aumentado exponencialmente su población en las últimas décadas (llegando a los 113.275 habitantes en 2018) tenía asignados siete escaños, mientras que la circunscripción insular de La Palma, con menos población de derecho (81.863 habitantes), tenía asignados ocho $^{22}$. A la hora de fijar un prorrateo para las circunscripciones insulares, la vigente DTP del EACan sigue optando por la asignación directa del mismo número de escaños a cada isla, con la única excepción de la circunscripción insular de Fuerteventura, a la que se asignan un escaño

19 Ya en el año 1997, el Pr. López Aguilar destacaba que «la extraordinariamente desigual relación que el sistema habría consagrado a la hora de conjugar el volumen de población de cada circunscripción insular con el correspondiente $n^{\circ}$ de escaños en el Parlamento, alcanzaría, en el peculiar subsistema político canario, niveles «escandalosos», López Aguilar, J. F., «Los problemas de constitucionalidad del sistema electoral canario (La insoportable permanencia de una Disposición Transitoria)», REDC, núm. 57, 1997, pp. 108-109.

20 Datos extraídos de las Cifras Oficiales de Población, actualizadas a fecha de 1 de enero de 2018, ofrecidos por el Instituto Canario de Estadística, disponibles en su web: http://www.gobiernodecanarias.org/istac/ temas_estadisticos/demografia/poblacion/cifraspadronales/E30245A.html

21 Esta jurisprudencia queda nítidamente reflejada en la STC 45/1992, de 2 de abril, que resuelve el Recurso de inconstitucionalidad promovido contra el art. 12.2 LEIB.

22 Datos extraídos de las Cifras Oficiales de Población, actualizadas a fecha de 1 de enero de 2018, ofrecidos por el Instituto Canario de Estadística, disponibles en su web: http://www.gobiernodecanarias.org/istac/ temas_estadisticos/demografia/poblacion/cifraspadronales/E30245A.html 
adicional. Por tanto, y debido a la gran dificultad de alcanzar un acuerdo político en otro sentido, la modificación del régimen electoral transitorio en relación al prorrateo de escaños entre islas no ha sido muy ambiciosa y lo único que hace es ajustarse al mandato, declarado en el art. 39.2.e) EACan, que impide que ninguna circunscripción insular tenga asignado un número inferior de diputados que otra que tenga menos población de derecho. En cualquier caso, la asignación de ocho escaños a la circunscripción insular de Fuerteventura implica la ruptura, poco significativa en términos cuantitativos, de dos de las tres paridades: las tres circunscripciones insulares de la provincia de Las Palmas suman 31 escaños y las cuatro circunscripciones insulares islas de la provincia de Santa Cruz de Tenerife suman 30; las circunscripciones de las cinco islas no capitalinas suman 31 escaños mientras que las dos islas capitalinas, suman 30; la única paridad que se mantiene ahora es la existente entre las dos islas capitalinas. Finalmente, la DTP del EACan asigna, en su apartado primero, los nueves escaños restantes a la circunscripción autonómica de Canarias. Se trata de una cifra modesta que sólo viene a atenuar parcialmente la enorme desproporcionalidad y la diferencia del valor relativo del voto entre circunscripciones insulares. Además, y como veremos en el último apartado, el tamaño de esta circunscripción va a imposibilitar, de facto, la entrada en el Parlamento de Canarias de fuerzas políticas que superen la barrera del 4 por ciento.

\section{LA BAJADA DE LAS BARRERAS ELECTORALES}

La reforma estatutaria operada mediante la Ley Orgánica 4/1996, de 30 de diciembre, supuso un aumento muy importante de las barreras de acceso al reparto de escaños: el porcentaje de votos que debían superar las listas de partido o coalición para obtener escaños en cada circunscripción insular pasó del 20 al 30\% de los votos válidos emitidos en la circunscripción, o alternativamente, del 3 al $6 \%$ de los votos válidos emitidos en el conjunto del archipiélago. El aumento de esta doble barrera electoral suscitó en su momento serias dudas de constitucionalidad que fueron denunciadas por el Defensor del Pueblo ante el Tribunal Constitucional $^{23}$. No obstante, en su STC 225/1998, el TC afirmaba que las barreras insulares vienen a «asegurar la presencia de fuerzas políticas mayoritarias en la circunscripción, pero minoritarias en el conjunto de la Comunidad Autónoma. Su objetivo no es, pues, el de excluir a las minorías, sino el de asegurar su presencia por razón del

23 La reforma, operada mediante la LO 4/1996, de 30 de diciembre, fue recurrida por el Defensor del Pueblo (Recurso de inconstitucionalidad 1324/1997). En su Sentencia 225/1998, de 26 de noviembre, el TC declara la constitucionalidad de las nuevas barreras electorales argumentando que «la valoración conjunta de este sistema de cláusulas limitativas en el acceso a los escaños no permite afirmar que sea contrario a la proporcionalidad exigida por el artículo $152.1 \mathrm{CE}$ » y que no le hacen perder «su capacidad para reflejar las principales opciones políticas existentes en la Comunidad Autónoma», STC 225/1998, F.J. $7^{\circ}$. 
territorio». El TC considera que las barreras insulares suponen un «correctivo» de la barrera electoral regional «en beneficio de la representación de cada isla, permitiendo la presencia en el Parlamento canario de candidaturas de fuerte implantación insular, aunque con escaso nivel de voto en su valoración global desde la óptica del voto emitido en el conjunto de la Comunidad Autónoma» ${ }^{24}$. En lo que respecta a la barrera del $6 \%$ que opera en el conjunto del archipiélago, el TC considera que «produce una diferencia de trato que pudiera tener una repercusión significativa desde el punto de vista del derecho de sufragio pasivo de los ciudadanos» pero, a pesar de situarse por encima de «esa pauta normalmente considerada como máxima que es el cinco por ciento» viene a declarar que se sitúa en el «límite de lo constitucionalmente tolerable ${ }^{25}$. A pesar de su constitucionalidad, lo cierto es que si acudimos al derecho electoral autonómico no existen, en ningún caso, barreras electorales tan restrictivas. En este sentido, en su clasificación de los sistemas electorales autonómicos, Oliver Araujo sitúa al canario en «el sexto (y máximo) grado del ranking de dureza de las barreras electorales autonómicas» ${ }^{26}$.

La grave incidencia de las dobles barreras electorales en los resultados de las elecciones autonómicas se podría ilustrar con muchísimos ejemplos. Especialmente llamativo es el caso de las elecciones de 2007 en la circunscripción de Gran Canaria en donde Nueva Canarias, tercera fuerza política con 46.303 votos ( $11,75 \%$ de los votos válidos), no consigue escaños por no superar ninguna de las barreras electorales —en el conjunto del archipiélago los sufragios obtenidos se quedan en el 5,43\%—; en esos mismos comicios, Coalición Canaria, que obtiene menos de la mitad de votos en la circunscripción de Gran Canaria (21.338 votos que representan el $5,41 \%$ de los válidamente emitidos), si obtiene un escaño ya que, en el conjunto del archipiélago, el número de votos obtenidos $(23,83 \%)$ superan la barrera regional del $6 \%$.

La reducción drástica de las barreras electorales era una demanda asimilada ya por todas las fuerzas políticas desde la propuesta de reforma del EACan que se aprueba en el año 2006 y que finalmente fue retirada de las Cortes por el Parlamento de Canarias. Como hemos visto en el apartado introductorio, este acuerdo también se refleja en la propuesta de reforma del EACan aprobada en marzo de 2015 que plantea la reducción a la mitad de cada una de las dos barreras de acceso. A su paso por la Comisión Constitucional del Congreso, y coincidiendo con la propuesta de reforma electoral aprobada por la Comisión de estudio del Parlamento de Canarias, la barrera electoral que debía operar en las circunscripciones insulares teniendo en cuenta los votos válidos emitidos en el conjunto del archipiélago se sube al $4 \%$, porcentaje que también se utiliza finalmente para fijar la barrera electoral de la nueva circunscripción autonómica.

24 STC 225/1998, F.J. $7^{\circ}$.

25 STC 225/1998, F.J. $5^{\circ}$

26 Oliver Araujo, J., Los sistemas electorales autonómicos, Barcelona: Ed. Institut d'Estudis Autonòmics, 2011, p. 377. 


\section{LA IMPLEMENTACIÓN DE LA REFORMA ELECTORAL EN LAS ELECCIONES DE MAYO DE 2019}

La reforma del EACan entra en vigor el 6 de noviembre de 2018, el mismo día de su publicación en el Boletín Oficial del Estado, sólo unos meses antes de la celebración de las elecciones autonómicas previstas para el 26 de mayo de 2016. Resultaba evidente que iba a ser muy complicado poner de acuerdo en este escaso margen de tiempo a los distintos grupos parlamentarios para elaborar y aprobar, por la mayoría cualificada requerida, la ley que debía regular el régimen electoral canario. Por lo tanto, las celebraciones de estos comicios debían regirse por una disposición transitoria cuya implementación debía ahora articularse jurídicamente. Con este objetivo, la Presidenta del Parlamento de Canarias dirige el 8 de noviembre de 2018 una consulta al Consejo Consultivo de Canarias, mediante el procedimiento de urgencia, sobre cuestiones referentes a la aplicación normativa del régimen transitorio del sistema electoral previsto en el nuevo EACan.

En este contexto, el Presidente del Gobierno de Canarias, sustentado en ese momento por Coalición Canaria y el Partido Popular, Fernando Clavijo, realiza unas desafortunadas declaraciones sembrado dudas respecto a la aplicación del nuevo régimen electoral sin que mediase su desarrollo legislativo ${ }^{27}$. Como no podía ser de otro modo, el Dictamen del Consejo Consultivo de Canarias 540/2018, de 28 de noviembre, que responde a la consulta del Parlamento de Canarias confirma lo evidente: el EACan «además de norma institucional básica de la CA, es norma jurídica cuyas prescripciones son directamente aplicables que vinculan tanto a los ciudadanos como a los poderes públicos». El legislador estatutario, continúa afirmando el Consejo Consultivo, «ha querido que en las próximas elecciones haya un régimen electoral distinto al que se deroga, sea porque se apruebe el de la DTP, sea porque se apruebe uno a través de la Ley del art. 39.2». Del mismo modo en que la anterior DTP había regido todas las elecciones autonómicas anteriores, la fuerza normativa de este nuevo régimen electoral transitorio, y su necesaria aplicación para las elecciones autonómicas de 2019, no podía ser objeto de ningún tipo de cuestionamiento jurídico ${ }^{28}$.

27 Consultar a este respecto las declaraciones publicadas por Europa-press el 18 de noviembre de 2018 disponibles en su web: https://www.europapress.es/nacional/noticia-clavijo-cree-reforma-electoral-canarias-debe-recogerse-ley-entrar-vigor-comicios-2019-20181018163958.html

28 En este mismo sentido se pronunciaban también López Aguilar y García Mahamut: «En tanto no sea aprobada la ley electoral (por 3/5) a la que se refiere el art. 39 EACan y la disp. trans. $1^{\mathrm{a}}$, esta última resulta autoaplicativa y directamente vinculante para todos los poderes públicos, y la inmediata aplicación y exigibilidad para la adjudicación de escaños en las próximas elecciones de 26 de mayo de 2019, en el n n y en la forma previstos por el nuevo EACan», López Aguilar J. F. y García Mahamut, R., «El nuevo Estatuto de Autonomía de Canarias: tercera generación. Hecho diferencial y nuevo sistema electoral», REDC, núm. 115, 2019, p. 41. También el Consejo Consultivo argumenta en su Dictamen 540/2018 que «de la misma manera que el régimen electoral transitorio vigente hasta la aprobación del nuevo Estatuto fue suficiente para regular las sucesivas elecciones que se celebraron bajo su vigencia, este nuevo régimen transitorio es bastante para ordenar la próxima consulta electoral autonómica porque, además de que, como se dijo, es clara la voluntad del 
Quedaba entonces por determinar si a la hora de aplicar las nuevas previsiones del EACan en la consulta electoral de 2019, el régimen jurídico vigente resultaba ser un parámetro normativo suficiente. El régimen electoral canario, además del art. 39 EACan y la DTP, se completa con la Ley 3/2007, de 20 de marzo, de Elecciones al Parlamento de Canarias, que regula aspectos accesorios del régimen electoral, con las previsiones de la Ley Orgánica 5/1985, de 19 de junio, del Régimen Electoral General (LOREG) que se aplican imperativamente, de acuerdo con su Disposición Adicional Primera, y también de manera supletoria ${ }^{29}$, así como el Decreto 99/2011, de 27 de abril que regula las condiciones de habilitación de locales, urnas, papeletas y otros materiales para la votación. Aunque en ninguna de estas normas encontrábamos referencias a la circunscripción autonómica, el Consejo Consultivo de Canarias considera en su Dictamen que a través de la «analogía, supletoriedad y principios generales del Derecho, es posible colmar las posibles lagunas que se puedan detectar como consecuencia del nuevo sistema electoral, cuya única novedad, a estos efectos de existir lagunas, la encontramos en la creación - y sus consecuencias- de una nueva circunscripción, la de ámbito autonómico, pues el resto de cuestiones (alteración de la distribución de escaños entre las circunscripciones insulares y las nuevas magnitudes de las barreras insulares) no las producen porque están expresamente resueltas. ${ }^{30} \mathrm{El}$ Consejo Consultivo da a entender que en caso de inactividad normativa (legislativa o reglamentaria) que regule la circunscripción autonómica, correspondería a la administración electoral, mediante instrucciones de la Junta Electoral de Canarias, resolver las dudas que pudiese suscitar su puesta en marcha ${ }^{31}$.

legislador estatutario de que se aplique si no hay nueva ley (aprobada por mayoría de tres quintos), se ha de partir de que el Ordenamiento Jurídico es único, completo y coherente, pues no solo contiene reglas que resuelven las eventuales antinomias que pudieran producir en su seno, sino que también es capaz de dar solución a todas las situaciones necesitadas de respuesta jurídica», Dictamen del Consejo Consultivo de Canarias 540/2018, de 28 de noviembre, fundamento IV.1.

29 La Disposición Adicional Primera de la LOREG determina que en «aplicación de las competencias que la Constitución reserva al Estado se aplican también a las elecciones a Asambleas Legislativas de Comunidades Autónomas convocadas por éstas, los siguientes artículos del Título I de esta Ley Orgánica: 1 al 42; 44; 44 bis; 45; 46.1, 2, 4, 5, 6 y 8; 47.4; 49; 50.1, 2 y 3; 51.2 y 3; 52; 53; 54; 58; 59; 60; 61; 62; $63 ; 65 ; 66 ; 68 ; 69 ; 70.1$ y $3 ; 72 ; 73 ; 74 ; 75 ; 85 ; 86.1 ; 87.2 ; 90 ; 91 ; 92 ; 93 ; 94 ; 95.3 ; 96 ; 103.2 ; 108.2 \mathrm{y}$ $8 ; 109$ a $119 ; 125$ a $130 ; 131.2 ; 132 ; 135$ a 152 . 3. Los restantes artículos del Título I de esta Ley tienen carácter supletorio de la legislación que, en su caso, aprueben las Comunidades Autónomas, siendo de aplicación en las elecciones a sus Asambleas Legislativas en el supuesto de que las mismas no legislen sobre ellos».

30 Dictamen del Consejo Consultivo de Canarias 540/2018, de 28 de noviembre, fundamento III.2.

31 En su Dictamen 540/2018, el Consejo Consultivo de Canarias considera que, para implementar el nuevo régimen electoral «no es necesario modificar formalmente ninguna Ley. Con meras decisiones de la Junta Electoral de Canarias, aplicando analógicamente la propia Ley de Elecciones al Parlamento de Canarias y directamente los arts. 19 y ss. de la LOREG, es posible asumir esas competencias y que las próximas elecciones al Parlamento de Canarias puedan realizarse con adecuación al régimen transitorio previsto en el Estatuto de Autonomía», Dictamen del Consejo Consultivo de Canarias 540/2018, de 28 de noviembre, fundamento IV.1. 
En cualquier caso, quedaban importantes cuestiones que debían ser resueltas para poner en marcha, con un mínimo de seguridad jurídica, la circunscripción autonómica. En lo que respecta a cómo se iba a ejercer el derecho de sufragio activo en cada una de las circunscripciones, el Parlamento de Canarias se cuestiona en la consulta dirigida al Consejo Consultivo si sería jurídicamente viable que los electores dispusieran de una única papeleta para elegir conjunta y obligatoriamente al mismo partido, federación, coalición o agrupación de electores en los dos tipos de circunscripción, sin posibilidad de optar por candidaturas de distinto signo político en cada circunscripción o abstener en alguna de ellas. Haciendo una interpretación de la legalidad inspirada en el sentido más favorable a la efectividad del derecho fundamental de sufragio, la respuesta negativa del Consejo Consultivo a esta cuestión resultaba también evidente ${ }^{32}$. En este sentido apuntaba también el informe sobre la articulación jurídica y puesta en marcha del sistema electoral contenido en la reforma del EACan elaborado por Pérez Sánchez y Fernández Esquer para Demócratas por el Cambio: «desde la perspectiva del sufragio activo, la posibilidad de obligar al ciudadano a votar la misma candidatura necesariamente en ambas circunscripciones, arrebatándole así la libertad de votar una opción política en una de estas circunscripciones y a otra distinta a la segunda de las circunscripciones, a nuestro juicio es claramente inconstitucional» ${ }^{33}$. Considerando que el elector debía contar con la posibilidad escoger entre distintas candidaturas en cada una de las circunscripciones, quedaba por resolver la cuestión de cómo articular ese doble voto. Una de las posibilidades, apuntada de manera implícita por el Parlamento de Canarias en su consulta, es que las candidaturas de los partidos a los dos tipos de circunscripción podían integrase en una única papeleta que permitiese dividir el voto ${ }^{34}$. Este tipo de sufragio, cono-

32 «Si lo que se nos cuestiona es si, durante la vigencia de la DTP y sin la nueva Ley del art. 39 EAC, es posible establecer que los electores dispusieran de una única papeleta para elegir conjunta y obligatoriamente al mismo partido, federación, coalición o agrupación de electores en los dos tipos de circunscripción (la autonómica y la insular), sin posibilidad de optar por candidaturas de distinto signo político en cada una de las dos circunscripciones o abstenerse en alguna de ellas, la respuesta debe ser negativa, pues el operador del Derecho debe aplicar, como se dijo, los mecanismos de hetero y auto integración para colmar las posibles lagunas mediante los mecanismos de la analogía, supletoriedad y los principios generales del Derecho (en este caso, el principio de interpretación más favorable a la efectividad de los derechos fundamentales)», Dictamen del Consejo Consultivo de Canarias 540/2018, de 28 de noviembre, fundamento IV.7.

33 Informe sobre la articulación jurídica y puesta en marcha del sistema electoral contenido en la reforma del EACAn, pp. 14-15, disponible en la web de Demócratas para el Cambio: https://reformaelectoralcanaria.files.wordpress.com/2018/11/informe-jurc3addico-aplicacic 3 b3n-rec-estatuto-versic3b3n-14-avales-para-registros.pdf. En este mismo sentido, se pronuncian López Aguilar y García Mahamut: «insertar el voto de la lista insular y de la lista regional en una sola papeleta y un urna común plantea dificultades respecto de su manejo por parte de cada elector/a y su posterior escrutinio, así como con respecto a su compatibilidad con el derecho de sufragio libre, igual, directo y secreto, puesto que podría producir un efecto impeditivo de la libertad de votar cada propia preferencia (y, en su caso, diferenciarla con voto discriminado): una en la lista insular y otra (idéntica o distinta) en la lista regional», López AgUILAR J. F. y GARCía Mahamut, R., op. cit., p. 42.

34 En la consulta dirigida al Consejo Consultivo, el Parlamento de Canarias formula la siguiente pregunta: « ¿Sería constitucional, estatutaria y legalmente viable que, en aplicación de las previsiones estatutarias 
cido como split-voting, es utilizado, por ejemplo, en las elecciones al Bundestag alemán. El Consejo Consultivo parece desechar esta posibilidad considerando que requeriría una compleja y detallada regulación legal y determina que «bajo la vigencia de la DTP, y sin esa regulación legal expresa, solo es posible garantizar el ejercicio del derecho de sufragio mediante la emisión del voto a través de dos papeletas y dos urnas distintas, en aplicación del principio de interpretación más favorable al ejercicio de los derechos fundamentales $»^{35}$. En este mismo sentido, se habían manifestado también López Aguilar y García Mahamut: «ante la eventual disyuntiva por la que cabría decidir entre la posibilidad de papeletas unitarias (con split voting, en su caso) y papeletas separadas (como sucede en el caso de la Camera dei Deputati italiana a partir de 1993), parece a nuestro juicio claro que la opción que, por principio, mejor facilita el derecho de sufragio es la doble papeleta y la doble urna» ${ }^{36}$.

Consultado sobre cuál debía ser el órgano de la administración electoral que asumiría las funciones de control en la nueva circunscripción autonómica, el Consejo Consultivo responde en su Dictamen que la Junta Electoral de Canarias, cuyo ámbito de actuación se extiende al conjunto del archipiélago, podría asumir las atribuciones relativas a la presentación, subsanación y proclamación de candidaturas, y verificación de papeletas y sobres electorales, que la Ley 7/2003 de Elecciones al Parlamento de Canarias asigna en sus arts. 17.1, 19.4, 20, apartados 2 y 3, y 24.2 , a las juntas electorales provinciales ${ }^{37}$.

Finalmente, el Gobierno de Canarias asume las recomendaciones contenidas en el Dictamen del Consejo Consultivo mediante la aprobación del Decreto 21/2019, de 18 de marzo, que modifica el Decreto 99/2011, de 27 de abril, que regula las condiciones de locales, urnas, papeletas, sobres y demás elementos materiales a utilizar en las elecciones al Parlamento de Canarias. En lo que respecta a la circunscripción autonómica, el art. 1 determina que cada mesa electoral debe contar con «una urna por cada circunscripción electoral, claramente identificada y precintada», mientras que los arts. 3 y 4 se refieren a las nuevas papeletas y sobres, de color amarillo, distintos de los utilizados para las circunscripciones insulares. Por último, en los anexos del Decreto se indican las características técnicas y condiciones de impresión de los nuevos sobres y papeletas de votación, así como de los restantes impresos electorales.

citadas, se estableciese que cada uno de los partidos, federaciones, coaliciones o agrupaciones de electores incluyan en una misma papeleta propia sus candidaturas a la circunscripción autonómica y a la correspondiente circunscripción insular? En tal caso, ¿sería constitucional, estatutaria y legalmente viable que: un elector quisiera votar a fuerzas políticas que únicamente presentasen candidatura en un solo tipo de circunscripción, cuando habría fuerzas políticas que presentan candidaturas en ambos tipos de circunscripciones?; un elector quisiese votar solo por una de las listas de la misma papeleta?; un elector quisiese votar a dos formaciones políticas distintas en cada una de las circunscripciones?», Dictamen del Consejo Consultivo de Canarias 540/2018, de 28 de noviembre, fundamento IV.9.

35 Dictamen del Consejo Consultivo de Canarias 540/2018, de 28 de noviembre, fundamento IV.9.

36 López Aguilar J. F. y García Mahamut, R., op. cit. p. 43.

37 Dictamen del Consejo Consultivo de Canarias 540/2018, de 28 de noviembre, fundamento IV.5. 


\section{ALGUNAS REFLEXIONES FINALES SOBRE LA INCIDENCIA DE LA REFORMA ELECTORAL EN LA PRÁCTICA}

Actualizado el régimen jurídico de las elecciones al Parlamento de Canarias, el Gobierno de Canarias emprende una tímida campaña informativa sobre las novedades de la reforma electoral. Esta campaña informativa no estuvo exenta de polémica ya que en una de sus cuñas radiofónicas se afirmaba que «cualquier canario podrá elegir directamente al candidato a futuro presidente de Canarias sin importar dónde resida, a través de la lista autonómica» ${ }^{38}$. Se trata, sin duda, de un grave error, denunciado por varias fuerzas políticas ante la Junta Electoral de Canarias, toda vez que el candidato a la Presidencia de Canarias puede ser cualquiera de los diputados, independiente de la circunscripción en la que haya sido elegido y del lugar que ocupase en las listas (art. 48.1 EACan). En cualquier caso, lo cierto es que buena parte de los partidos políticos más representativos, entre ellos PSOE, Coalición Canaria y Nueva Canarias, sitúan a sus candidatos a la Presidencia de Canarias en la cabeza de las listas que presentan a la circunscripción autonómica. Sin embargo, otros partidos políticos, como Podemos, integran a sus candidatos en las listas que se presentan en las circunscripciones insulares en donde, de acuerdo con sus cálculos electorales, concentran más votos y les será más fácil obtener escaño.

En lo que respecta a los resultados de las elecciones del 26 de mayo de 2019 debemos constatar que a la postre posibilitaron la alternancia política, después de más de veinticinco años, en el Gobierno de Canarias, sustentado ahora por la mayoría parlamentaria que se forma tras el «Pacto de las Flores», suscrito entre el PSOE, Nueva Canarias, Sí Podemos Canarias y la Agrupación Socialista Gomera. En cualquier caso, y como comprobaremos a continuación, no parece que la reforma electoral fuese determinante para la consecución de este cambio político.

En lo respecta al ejercicio de sufragio mediante el doble voto, y de acuerdo con los datos ofrecidos por el Parlamento de Canarias ${ }^{39}$, los electores no discriminan de manera significativa el voto que emiten en cada una de las circunscripciones. La participación en el conjunto de las circunscripciones insulares ( $52,59 \%$ de los electores) y en la circunscripción autonómica (52,54\%) es prácticamente idéntica; y tampoco existen variaciones muy significativas entre el número de votos que cada partido obtiene en el conjunto de las circunscripciones insulares y el número de votos que reúne en la circunscripción autonómica ${ }^{40}$.

38 Respecto a la difusión de la campaña informativa del Gobierno de Canarias consultar la noticia «El Gobierno de Canarias confunde a los votantes con una campaña sobre el nuevo sistema electoral» publicada en Eldiario.es, el 4 de marzo de 2019, disponible en la web: https://www.eldiario.es/canariasahora/politica/ Gobierno-Canarias-confunde-votantes-electoral_0_874213341.html

39 Los datos de participación en las elecciones al Parlamento de Canarias han sido extraídos de las web institucional: https://www.parcan.es/elecciones/resultados.py/2019/

40 El PSC-PSOE obtuvo 258.255 votos $(28,56 \%)$ en el conjunto de las circunscripciones insulares y $264.221(29,22 \%)$ en la circunscripción autonómica; El PSC-PSOE obtuvo 258.255 votos (28,56\%) en el conjunto de las circunscripciones insulares y 264.221 (29,22\%) en la circunscripción autonómica; Coalición 
En los comicios de 2019, la primera fuerza política en número de votos tanto en el conjunto de las circunscripciones insulares como en la circunscripción autonómica, el PSC-PSOE, es la que obtiene un mayor número de escaños. No obstante, los resultados obtenidos en la circunscripción autonómica no refuerzan especialmente a esta fuerza política. De hecho, si se hubiese mantenido el sistema electoral anterior, sin la circunscripción autonómica, la relación de fuerzas entre los grupos parlamentarios que apoyan al gobierno y los que se sitúan en la oposición en la actual legislatura sería prácticamente idéntica, lo que hubiese posibilitado igualmente el cambio político. Si descontamos los diputados de la circunscripción autonómica y el octavo diputado elegido en la circunscripción de Fuerteventura, de Coalición Canaria, el Gobierno podría estar sustentado por la mayoría de 32 diputados (en lugar de los 37 diputados de un parlamento de 70 escaños).

Tal y como puede comprobarse en la Tabla 2, la reducción de las barreras electorales en estos comicios tampoco repercute significativamente en los resultados. Ciudadanos, el partido político que había quedado fuera del Parlamento tras las elecciones de 2015 por no superar la barrera electoral del 6\%, supera ahora este umbral tanto en el conjunto de las circunscripciones insulares como en la circunscripción autonómica. No obstante, el 6,87\% de los votos que obtiene ciudadanos en la circunscripción no son suficientes para obtener escaño, por lo que la barrera que de facto opera en esta circunscripción, de tamaño medio, se sitúa bastante por encima del $4 \%$.

Los diputados elegidos en mayo de 2019 deben cumplir con el mandato contenido en la DTP y elaborar, antes de noviembre de 2021, la ley que regule el régimen electoral canario. Aunque el incumplimiento de este mandato no tiene consecuencia jurídica alguna, el legislador canario debería comprometerse decididamente con este objetivo, partiendo, tal vez, de las bases que ahora propone la DTP y que han suscitado un amplio acuerdo político. En cualquier caso, si lo que se pretende es reducir sensiblemente la desigualdad del valor relativo del voto entre circunscripciones insulares debería plantearse la posibilidad de adoptar un nuevo modelo de prorrateo de escaños que supere, de una vez por todas, el que se ha venido inspirando en la triple paridad (corregida ahora por la asignación de un escaño adicional a la circunscripción de Fuerteventura). Tal vez la opción más

Canaria obtuvo 196.080 votos $(21,68 \%)$ en el conjunto de las circunscripciones insulares y $209.150(23,13 \%)$ en la circunscripción autonómica; el Partido Popular obtuvo 135.722 votos $(15,01 \%)$ en el conjunto de las circunscripciones insulares y $130.617(14,45 \%)$ en la circunscripción autonómica; Nueva Canarias obtuvo 80.891 votos $(8,94 \%)$ en el conjunto de las circunscripciones insulares y $82.980(9,18 \%)$ en la circunscripción autonómica; Sí Podemos Canarias obtuvo 78.532 votos $(8,68 \%)$ en el conjunto de las circunscripciones insulares y $76.433(8,45 \%)$ en la circunscripción autonómica; Ciudadanos obtuvo 65.854 votos $(7,28 \%)$ en el conjunto de las circunscripciones insulares y 62.115 (6,87\%) en la circunscripción autonómica; y Agrupación Socialista Gomera obtuvo 6.222 votos $(0,69 \%$ en el total de votos del conjunto de circunscripciones insulares), todos ellos en la circunscripción de La Gomera, la única a la que concurrió.

Los resultados completos de las elecciones al Parlamento de Canarias de mayo de 2019 pueden consultarse en la web institucional: https://www.parcan.es/elecciones/resultados.py/2019/ 
sensata sería la asignación inicial de un número concreto de escaños a cada circunscripción insular y el prorrateo del resto de escaños reservados a las circunscripciones insulares atendiendo a criterios demográficos en cada una de las convocatorias electorales, tal y como ocurre en el caso de las elecciones al Congreso de los Diputados. La futura legislación electoral canaria también debería consolidar la circunscripción autonómica asignándole un número suficiente de escaños para que opere efectivamente el principio constitucional de representación proporcional.

\section{$* * *$}

TITLE: Electoral reform in the Canary Islands: origin, scope, implementation and impact

ABSTRACT: The reform of the Statute of Autonomy of the Canary Islands, approved by Organic Law 2/2018, of 5 November, has finally changed the complex regime of the regional parliamentary elections, persistently demanded by constitutional scholars and civil society. This reform affects the dimension of the Parliament of the Canary Islands, involves the creation of a new regional constituency, which coexists with the traditional island constituencies, alters the apportionment of seats and drastically reduces the electoral thresholds. Considering that the new electoral regime must be applied in the regional elections beld in May 2019, we offer an analysis of the legal instruments used for its implementation. Finally, and taking into account the results of the aforementioned elections, we will present some reflections on the modest practical impact of the reform.

Resumen: La reforma del Estatuto de Autonomía de Canarias, aprobada mediante la Ley Orgánica 2/2018, de 5 noviembre, ba supuesto la modificación de las bases del particular sistema electoral canario, insistentemente demandada por la doctrina constitucional y desde amplios sectores de la clase política y la sociedad civil canaria. Esta reforma incide en la dimensión del Parlamento de Canarias, supone la creación de una nueva circunscripción de ámbito autonómico, que coexiste con las tradicionales circunscripciones insulares, altera el prorrateo de escaños entre circunscripciones y reduce drásticamente las barreras electorales. Considerando que las nuevas bases del régimen electoral canario debian ser aplicadas necesariamente en las elecciones autonómicas celebradas en mayo de 2019, ofrecemos un análisis de los instrumentos jurídicos que, a falta de una ley electoral de desarrollo, fueron utilizados para su implementación. Finalmente, y atendiendo a los resultados de las mencionadas elecciones, presentaremos algunas reflexiones sobre la modesta incidencia práctica de la reforma.

KEY WORDS: Electoral systems, electoral law, regional elections, Parliament of the Canary Islands.

Palabras Clave: Sistemas electorales, derecho electoral, elecciones autonómicas, Parlamento de Canarias.

FECHA DE RECEPCIÓN: 21.12.2019

FECHA DE ACEPTACIÓN: 19.02.2020 
